\title{
A Numerical Method for Calculating Stellar Occultation Light Curves from an Arbitrary Atmospheric Model
}

\author{
D. M. Chamberlain ${ }^{1,2}$ AND J. L. Elliot ${ }^{3}$ \\ Department of Earth, Atmospheric and Planetary Sciences, Massachusetts Institute of Technology, \\ Cambridge, Massachusetts 02139-4307 \\ Electronic mail: dawn@cam.cornell.edu, jim@astron.mit.edu \\ Received 1996 August 29; accepted 1997 June 5
}

\begin{abstract}
We present a method for speeding up numerical calculations of a light curve for a stellar occultation by a planetary atmosphere with an arbitrary atmospheric model that has spherical symmetry. This improved speed makes least-squares fitting for model parameters practical. Our method takes as input several sets of values for the first two radial derivatives of the refractivity at different values of model parameters, and interpolates to obtain the light curve at intermediate values of one or more model parameters. It was developed for small occulting bodies such as Pluto and Triton, but is applicable to planets of all sizes. We also present the results of a series of tests showing that our method calculates light curves that are correct to an accuracy of $10^{-4}$ of the unocculted stellar flux. The test benchmarks are (i) an atmosphere with a $1 / r$ dependence of temperature, which yields an analytic solution for the light curve, (ii) an atmosphere that produces an exponential refraction angle, and (iii) a small-planet isothermal model. With our method, least-squares fits to noiseless data also converge to values of parameters with fractional errors of no more than $10^{-4}$, with the largest errors occurring in small planets. These errors are well below the precision of the best stellar occultation data available. Fits to noisy data had formal errors consistent with the level of synthetic noise added to the light curve. We conclude: (i) one should interpolate refractivity derivatives and then form light curves from the interpolated values, rather than interpolating the light curves themselves; (ii) for the most accuracy, one must specify the atmospheric model for radii many scale heights above half light; and (iii) for atmospheres with smoothly varying refractivity with altitude, light curves can be sampled as coarsely as two points per scale height.
\end{abstract}

\section{INTRODUCTION}

Since Baum and Code's observations of a stellar occultation by Jupiter in 1952 (Baum and Code 1953), the analysis of occultation data to learn about the structure of planetary atmospheres has developed along two lines: numerical inversion (Kovalevsky and Link 1969; Wasserman and Veverka 1973; French et al. 1978) and model fitting (Baum and Code 1953; Elliot and Young 1992). In principle, for atmospheres in hydrostatic equilibrium, the inversion approach will yield the temperature profile, as well as pressure and number density profiles (if one knows the planetary gravity and mean molecular weight-which effectively means knowing the composition). However, small uncertainties in the initial conditions cause significant errors in the results until the inversion calculation has been carried out for several scale heights (Wasserman and Veverka 1973; French et al. 1978; Roques et al. 1994). By then, however, the occultation light-curve flux is low and errors in the zero flux level can significantly affect the results. Noise in the light curve also adds spurious structure to the inversion results. In spite of these difficulties, inversion is

\footnotetext{
'Also Department of Mathematics, Massachusetts Institute of Technology. ${ }^{2}$ Now at Center for Applied Mathematics, Comell University, Ithaca, NY 14853-3801.

${ }^{3}$ Also at Department of Physics, Massachusetts Institute of Technology, and Lowell Observatory, Flagstaff, AZ 86001-4499.
}

the best method of analysis when one does not have an $a$ priori model of the atmospheric structure.

The modeling approach has been limited to isothermal atmospheres (Baum and Code 1953) and atmospheres with power-law thermal gradients (Elliot and Young 1992, hereafter EY92). However, our theoretical understanding of atmospheric structure has progressed far beyond just estimating a scale height, and the thermal gradients in atmospheres would not necessarily follow a power law. For example, physical models of Triton's atmosphere (Krasnopolsky et al. 1993; Strobel and Summers 1995) include the physical effects of electron heating, solar radiative heating of $\mathrm{CH}_{4}$, thermal conduction, and radiative cooling by $\mathrm{CH}_{4}$ and $\mathrm{CO}$. With a stellar occultation data set of high quality (e.g., Olkin 1996), one can hope to test the validity of these models, and-for those models found to be valid-to use the data to estimate values of atmospheric parameters, such as the $\mathrm{CH}_{4}$ and $\mathrm{CO}$ fractions in the atmosphere.

To carry out these analyses, one would like to fit an appropriate atmospheric model to the occultation light curve by least squares, in order to find the most likely values of the model parameters and their errors. Because of the complexity of the atmospheric models, a numerical approach is required: for each set of model parameters, a model light curve must be calculated, and for each parameter being fit, one must calculate a numerical derivative for each point on the light curve by incrementing the parameter and calculat- 
ing another model light curve. Each time the physical model is recalculated (e.g., Strobel and Summers 1995), this process involves equations that can only be solved by numerical iteration to find self-consistent values, which, at present, takes several hours (Strobel, personal communication). Hence computing a light curve and its numerical derivatives from such a model for each parameter fit can involve an enormous amount of computing time for each set of parameters. This computing time can make least-squares fitting for the best parameter values cumbersome at best, and often impractical.

In this paper we present a method for speeding up the process of calculating light curves from complicated atmospheric models by interpolating a grid of precalculated models. In calculating the light curve, we include geometrical effects appropriate to small planets (EY92). Our model is thus applicable to occulting bodies like Pluto and Triton as well as to larger planets. We also allow for the effects of extinction, though we do not present results of tests that include it. We start with one or more profiles of values for the first two radial derivatives of the refractivity at radii spanning the region probed by the occultation, and we interpolate between these values to calculate a model light curve for some set of parameters. We present a method for interpolating between points along a single refractivity profile to make a model light curve from a single set of parameters and for interpolating between models with differing values of parameters to make light curves at arbitrary intermediate values of the model parameters.

In deriving the equations for converting a refractivity profile into a light curve, we closely follow Sec. 2 of EY92. As they did, we use the geometrical optics approximation and make some simplifying assumptions: (i) the light received at any time comes from only one point on the planetary limb, (ii) the atmosphere is spherically symmetric. These assumptions are not critical to our method, however, and we make them only to simplify the numerical computations presented here.

\section{THE MODEL}

\subsection{Analytic Basis}

We assume that parallel, monochromatic light rays are incident on a spherically symmetric planetary atmosphere from the left in Fig. 1. The observer's plane is perpendicular to the path of the light rays. In this plane, $y$ is a radial coordinate measuring the observer's position relative to the point through which a line connecting the occulted star and the center of the planet would pass. EY92 used a coordinate $\rho=|y|$. Using $y$ instead of $\rho$ enables us to more easily include contributions to the flux from both the near and far limbs (although in this paper we shall be working with only the near-limb flux). We refer to $y$ as a function of $r$, which marks the position of arrival in the observer's plane of a light ray with a radius of the closest approach to the planet of $r$. We also refer to $y$ as a function of $t$, where $y(t)$ marks the position of the observer in the plane as a function of time. This function can be determined from the geocentric planetary ephemeris and the motion of the observer

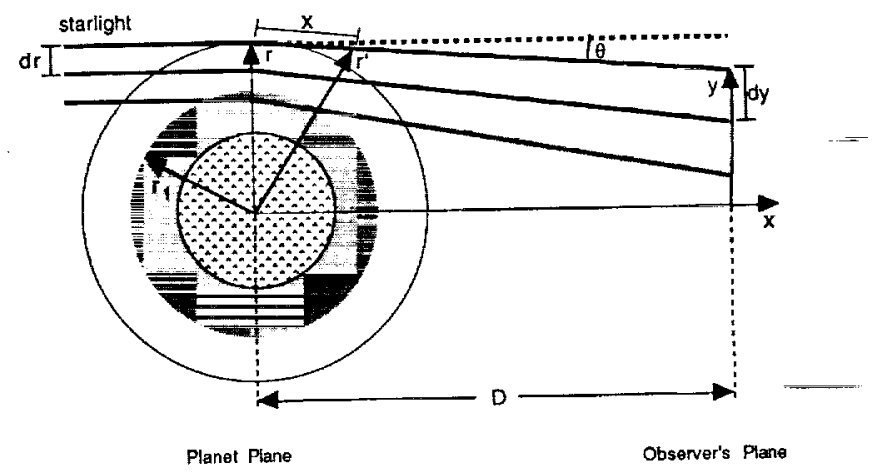

FIG. 1-Stellar occultation by a planetary atmosphere. Starlight encounters a planetary atmosphere and is bent by the refractivity gradient in the atmosphere. Since the refraction increases exponentially with depth into the atmosphere, refraction causes two neighboring rays to be bent by a slightly different angle, and this differential separation increases by an amount proportional to distance from the planet. This effect causes the star to $\operatorname{dim}$ as seen by a distant observer (after EY92).

relative to the center of the Earth. The refraction angle, $\theta(r)$, is defined in the usual sense to be positive above the axis, so refraction by the atmosphere produces negative values.

With these assumptions, the flux from the occulted star can be broken down into three terms: (i) the differential refraction of the light rays at different depths in the atmosphere, (ii) the partial focusing of the light rays in the plane perpendicular to the path of the ray, and (iii) the extinction of the light due to absorption in the atmosphere. From EY92, this yields the following equation for the flux, $\zeta$, as a function of $r$ :

$$
\zeta(r)=\frac{1}{\mid 1+\underset{\text { differential refraction }}{D[d \theta(r) / d r] \mid}} \frac{1}{\mid 1+\underset{\text { Focusing }}{D \theta(r) / r \mid} \exp \left[-\tau_{\text {obs }}(r)\right]}
$$

where $\tau_{\text {obs }}(r)$ is the optical depth along the path of the ray, $D$ is the distance between the occulting body and the observer, and $\theta(r)$ is the angle through which a light ray is refracted as a function of the closest approach, $r$, to the center of the occulting body. If we made no assumptions about the refractivity in the atmosphere, we would need to include a factor of one over the index of refraction in the calculation of $\theta(r)$ (Born and Wolf 1964). To simplify the calculation we assume that the refractivity $\nu \ll 1$ and that $|\theta| \ll 1$ throughout the atmosphere. Under this assumption, the following approximations are valid:

$$
\theta(r)=\int_{-\infty}^{\infty} \frac{r}{r^{\prime}} \frac{d \nu\left(r^{\prime}\right)}{d r^{\prime}} d x
$$

and

$$
\frac{d \theta(r)}{d r}=\int_{-\infty}^{\infty}\left\{\frac{x^{2}}{\left(r^{\prime}\right)^{3}} \frac{d \nu\left(r^{\prime}\right)}{d r^{\prime}}+\frac{r^{2}}{\left(r^{\prime}\right)^{2}} \frac{d^{2} \nu\left(r^{\prime}\right)}{d r^{\prime 2}}\right\} d x
$$

where $x$ is a coordinate along the path of the ray measured from its point of closest approach and $r^{\prime}$ is the radial distance from the planet along the path of the ray (see Fig. 
1). The variables $r, r^{\prime}$, and $x$ satisfy the relation $r^{\prime 2}=r^{2}+x^{2}$.

In this paper we shall be referring to $\phi_{\mathrm{cyl}}(y)$, the unfocused stellar flux from one limb that is not affected by extinction. The subscript "cyl" reminds us that this is the flux that would be seen refracted by a cylindrical atmosphere (one with no focusing in the plane perpendicular to the ray path). The range of $\phi_{\text {cyl }}(y)$ corresponds to a normalized stellar flux, which equals 1.0 when the starlight is unaffected by the atmosphere and 0.0 when the stellar flux is totally occulted. Since focusing and extinction are not included, our equation for $\phi_{\text {cyl }}(y)$ includes only the first term of Eq. (1):

$$
\phi_{\text {cyl }}(y)=\frac{1}{\left|1+D \frac{d \theta(r)}{d r}\right|},
$$

where $y$ is related to $r$ by

$$
y=r+D \theta(r) .
$$

The derivatives of the refractivity required to calculate $\theta(r)$ and $d \theta(r) / d r$ are supplied by the atmospheric model. Note that only the first and second derivatives of the refractivity are needed to calculate the light curve, and not the refractivity itself.

For atmospheres with $\nu(r)$ monotonically decreasing with $r$, the derivative in Eqs. (1) and (4) will always be positive, and $\phi_{c y l}$ will be finite. However, this condition does not hold for all atmospheric models (and all real atmospheres), so that $\phi_{\text {cyl }}$ can have infinite values when the denominator in Eq. (4) equals zero. We have not addressed this problem in the method presented here. This problem could be addressed in the future by integrating $\phi_{\text {cyl }}(y)$ over a small interval $\Delta y$. This integral will be finite since the total light flux is finite. Also, the proposed integration procedure for treating points where Eq. (4) becomes infinite would correspond to a real light curve, since any observed light curve is always integrated (by the data recording equipment) over some finite interval of distance within the occultation shadow.

\subsection{Numerical Implementation}

The next step is to evaluate the equations developed in the previous section using only a set of values of the first two derivatives of $\nu\left(r^{\prime}\right)$ at discrete radii. In order to use Eq. (1) to calculate the flux, we need to put $d \nu\left(r^{\prime}\right) / d r^{\prime}$ and $d^{2} \nu\left(r^{\prime}\right) / d r^{\prime 2}$ into integrable forms. We do this by interpolating between the values we have for these quantities. The interpolation functions are only valid out to $x_{\max }$, where

$$
x_{\max }^{2}=r_{\max }^{2}-r^{2} .
$$

Hence, we approximate $\theta$ and $d \theta / d r$ by numerically integrating Eqs. (2) and (3) out to $\pm x_{\max }$. Since the angle of bending in atmospheres falls off exponentially with increasing $r$, in principle we can choose $x_{\max }$ so that the contribution to the integral beyond it is as small as desired.
To calculate a model light curve that we can compare with occultation data we need the flux, and hence $\theta$ and $d \theta / d r$, as a function of time. Since we have $\theta$ and $d \theta / d r$ as functions of radius, we want to find the radius as a function of time. We do this in two steps. First we choose a set of values of $r$ (we used equally spaced points for simplicity) and use our formula for $\theta$ in Eq. (5) to make a list of $(r, y)$ pairs. We use these pairs to make an interpolation function for $r(y)$. Next we determine the function $y(t)$ from the details of the occultation we observed. For small planet occultations, EY92's Eq. (5.1) is often appropriate. For larger planets and/or longer occultations, the linear approximation for $y(t)$ may not be adequate, and we must calculate it from the planetary ephemeris and the motion of the observer for each desired time, $t$ (Elliot et al. 1993).

Once we have $r(y)$ and $y(t)$, we are able to calculate the radius corresponding to each time in which we are interested. We can then use our equations for $\theta$ and $d \theta / d r$ to calculate the first two terms in Eq. (I) at these radii, which is the flux we are interested in (see Fig. 2).

\subsection{Interpolations}

An alternative method for calculating light curves for parameter values between those of the given refractivity derivative profiles would be to calculate the light curve for each refractivity profile, then interpolate between the light curves themselves, rather than interpolating the refractivities. However, interpolation assumes that the function being approximated is smooth; when there are discontinuities or sharp changes in the function, interpolation can introduce large errors. Light curves can have discontinuities when, for example, the surface of the planet moves in front of the occulted star. Geometrical effects near the center of the shadow can also cause abrupt changes in the light curve, such as the "central flash" (Elliot et al. 1977). Since the derivatives of the refractivity change more smoothly, the better course is to interpolate these derivatives and then calculate the light curve (rather than interpolating a grid of light curves). Another reason for interpolating the derivatives of the refractivity is that if one has data from several stations for the same occultation by a planet with a spherically symmetric atmosphere, one need calculate only one interpolation function for the refractivity derivatives (since the refractivity as a function of planetary radius will be the same for all stations). If one interpolated the light curves instead, different interpolation functions would be needed for each station, since the light curves will look very different at stations that probed the atmosphere at different minimum distances from the center of the occultation shadow.

The interpolation functions we made for the derivatives of the refractivity profiles needed to be multidimensional. Many methods for making such interpolation functions require a rectangular grid of points as inputs. Rather than requiring that each profile have values for the derivatives at the same set of radii, we chose to make the interpolation functions in two steps. First, we made one-dimensional interpolation functions for the derivatives from each profile. 


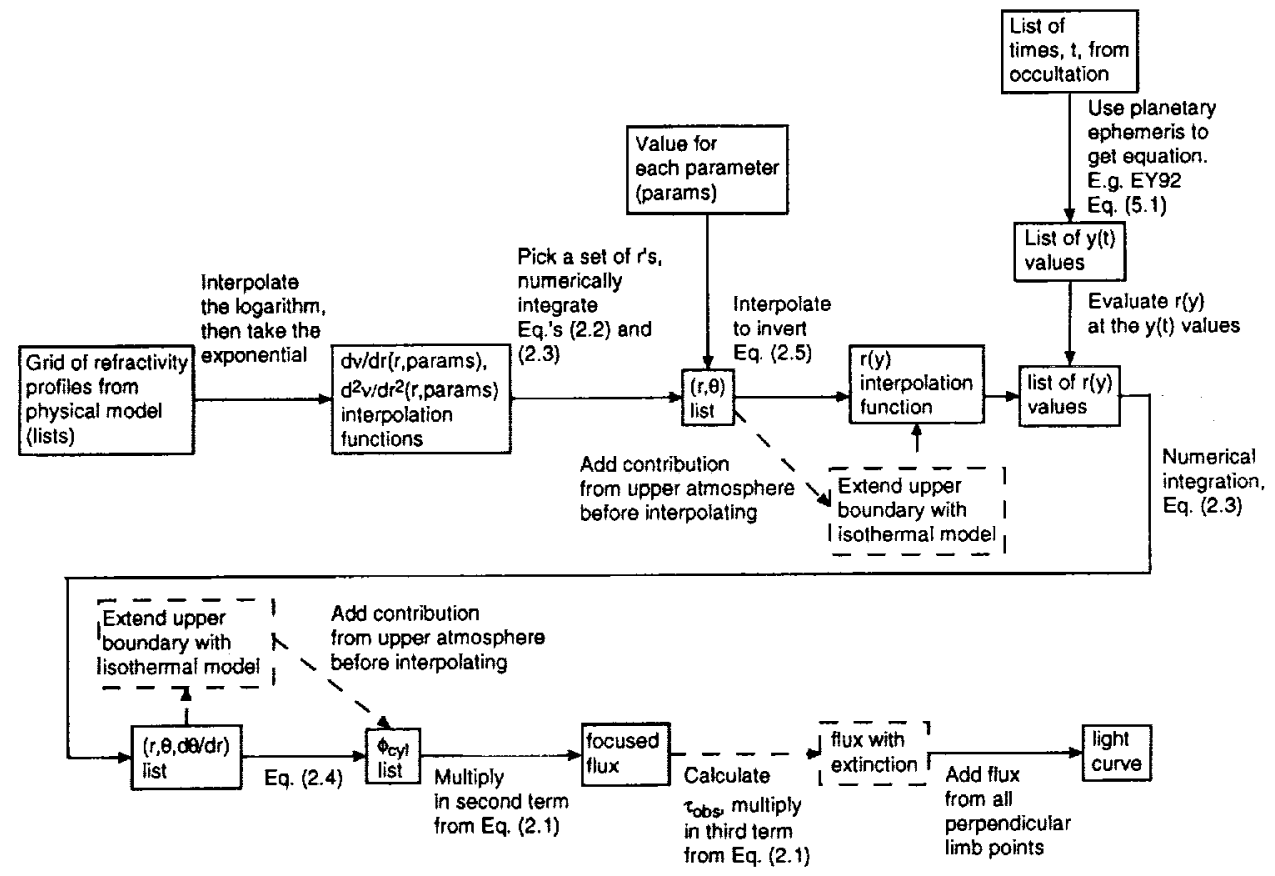

FIg. 2-Calculation flow chart. This diagram summarizes the steps in our method for numerically calculating the light curve from refractivity derivative profiles. The arrows represent procedures and the solid boxes represent the input to these procedures and their results. Dotted lines indicate optional steps.

We then used these interpolation functions to calculate the derivatives of the refractivity for each set of parameter values at a common set of radii, thereby giving us a rectangular grid of points that we could use to make our multidimensional interpolation functions.

\subsection{Boundary Conditions at Large Radii}

It is often necessary to fit a long section of the light curve when the star is not occulted in addition to the occultation event itself in order to establish the full scale brightness of the star. With the method described above, fitting a long upper baseline requires that values of the refractivity be calculated out to very large radii. If calculating such values is time consuming, we can make a further approximation for the flux beyond $r_{\max }$ to extend the method with less computation time. We reject the simplest possible approximation of setting the flux to 1.0 beyond $r_{\max }$ because this introduces a discontinuity in the flux, which can interfere with least-squares fitting of these models to occultation data. Instead, we approximate the values at the ends using the model described in Secs. 3 and 4 of EY92, calculating their model to only first order and assuming that the outer atmosphere is isothermal. A detailed explanation of how to do this is presented by Chamberlain (1996).

\subsection{Software Implementation}

We chose to implement our method as a package in Mathematica ${ }^{\text {TM }} 2.2$ (Wolfram 1991) on a Power Macintosh $8100 / 80$ running a remote kernel on an HP9000 735/125. Mathematica ${ }^{\mathrm{TM}}$ compromised some computing speed for ease of development. The speed penalty could be ameliorated by implementing our method either fully in the $\mathrm{C}$ programming language, or in something like Mathematica's MathLink ${ }^{\mathrm{TM}}$ protocol, which allows calls to $\mathrm{C}$ programs from Mathematica ${ }^{\mathrm{TM}}$ notebooks. From tests comparing the speed of C programs to that of Mathematica ${ }^{\mathrm{TM}}$ notebooks, we estimate that our method would run about 100 times faster on the same machine if we implemented it in $\mathrm{C}$ rather than in Mathematica TM.

To increase speed as much as possible within Mathematica $^{\mathrm{TM}}$, we used Mathematica's internal numerical integration and interpolation functions. Mathematica ${ }^{\mathrm{TM}}$ uses piece-wise continuous polynomials to interpolate and adaptive quadrature to numerically integrate. Table I details what we interpolated and what order polynomials we used.

\section{TESTS}

We performed several tests of our method. We tested its accuracy for calculating single light curves, and then for multiple light curves (as would be needed for least-squares fitting of atmospheric models). Also we note the computing time for a typical calculation.

\subsection{Single Light Curves}

The three model atmospheres used in our accuracy tests were: (i) an analytic model atmosphere, for which we can specify a power-law temperature profile that corresponds to a light curve that can be written with familiar functions (Eshleman and Gurrola 1993); (ii) a tightly bound atmosphere, close to that originally given by Baum and Code (1953); (iii) a loosely bound atmosphere from the smallplanet model of EY92. 
TABLE 1

Summary of Interpolated Functions

\begin{tabular}{|c|c|c|c|c|}
\hline $\begin{array}{l}\text { Function } \\
\text { interpolated }\end{array}$ & $\begin{array}{l}\text { Order of } \\
\text { interpolation }\end{array}$ & $\begin{array}{l}\text { Interpolated } \\
\text { variable }\end{array}$ & $\begin{array}{l}\text { Spacing of Points } \\
\text { (per scale height) }\end{array}$ & Comments \\
\hline $\begin{array}{l}d \nu\left(r,\left\{p_{i}\right\}\right) / d r \\
d^{2} \nu\left(r,\left\{p_{i}\right\}\right) / d r^{2}\end{array}$ & 3 & $r$ & $2-10$ & $\begin{array}{l}\text { Interpolated the natural log of the function } \\
\text { Included derivatives for } d \nu(r) / d r\end{array}$ \\
\hline $\begin{array}{l}d \nu\left(r,\left\{p_{i}\right\}\right) / d r \\
d^{2} \nu\left(r,\left\{p_{i}\right\}\right) / d r^{2}\end{array}$ & 3 & $\stackrel{r}{\left\{p_{i}\right\}^{\mathrm{a}}}$ & $\begin{array}{c}30-40 \\
\ldots\end{array}$ & $\begin{array}{l}\text { Interpolated the natural log of the function } \\
\text { Spacing of points varied }\end{array}$ \\
\hline$r(y)$ & 3 & $y$ & 50 & Spacing of points varied \\
\hline
\end{tabular}

${ }^{a}\left\{p_{i}\right\}$ represents one or more parameters of the atmospheric model.

"Optimum spacing of atmospheric parameter values is highly dependent on the parameter.

\subsubsection{Analytic Atmosphere}

To describe the degree to which a planetary atmosphere is bound, we use the parameter $\lambda_{h}$, which, as defined by Eq. (3.9) of EY92, is equal to the ratio of the gravitational potential to $k T$ at the half-light radius, $r_{h}: \lambda_{h}=G M \mu m /$ $k T r_{h}$, where $G$ is the gravitational constant, $M$ is the mass of the planet, $\mu$ is the molecular weight, $m$ is the atomic mass unit, $k$ is Boltzmann's constant, and $T$ is the temperature. We made a refractivity profile using the model specified in the Appendix (A.1) with $\lambda_{h}=100$ and $r_{h}=1500$ $\mathrm{km}$ and compared the light curve we calculated with our method to a light curve calculated analytically with the model. The light curve and the differences between our method and the model light curve are shown in Figs. 3 and 4.

The difference curve shows a maximum difference of less than $10^{-7}$, which is far smaller than noise levels in real occultation data. However, it also shows two discontinuities. To investigate the origin of the discontinuities, we calculated the first and second differences of the flux from both the model and our method for the eight points surrounding each discontinuity. These differences showed that the second discontinuity appears in the flux from our method, but not in the flux from the model. The first discontinuity did not seem to appear in either set of numbers, so we made a light curve from radii that were spaced 20 times closer together around both discontinuities and found that the second one remained a discontinuity, but the first

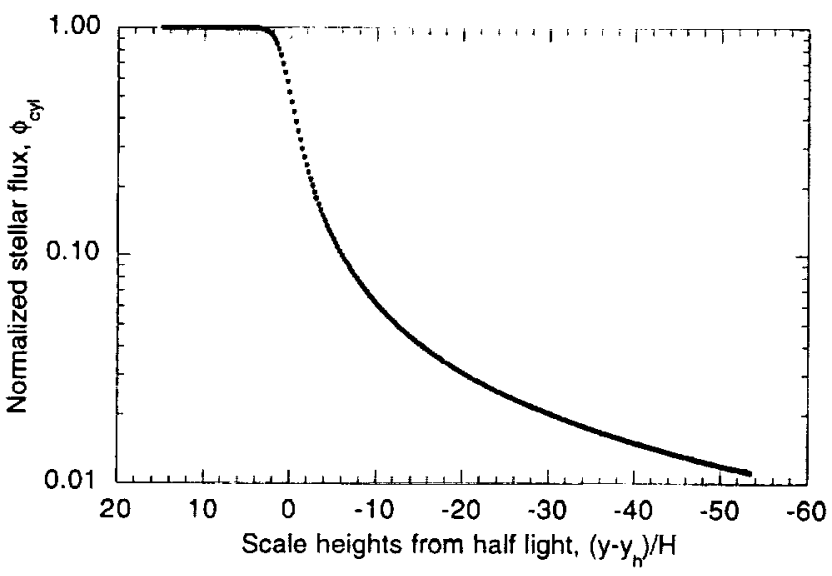

FIG. 3-Sample light curve. This light curve was calculated with the analytic benchmark model according to the prescription given in Appendix (A.1), for $\lambda_{h}=100$ and $r_{h}=1500$. one clearly showed points spanning the gap of what had looked like a discontinuity in the original test. Both of these sharp changes are most likely due to numerical limitations in our method.

\subsubsection{Tightly Bound Atmosphere}

Since the first-order structure of an atmosphere is usually exponential rather than a power law, the second benchmark model is one in which the refraction angle is strictly an exponential function of radius (see Appendix A.2). We ran a series of tests with this model. When we compared our method to the first-order approximation, $\nu_{1}(r)$, we found that tests with $\lambda_{h}=4 \times 10^{7}$ showed the smallest differences between our method and results calculated from the exponential refraction model, with larger differences at both larger and smaller values of $\lambda_{h}$ (see Fig. 5). The differences for values larger than $4 \times 10^{7}$, shown in Fig. 6, were randomly scattered around zero, while differences for smaller values followed a smooth curve. This implies that the differences were due to round off at larger values and the first-order approximation at smaller values.

When we re-ran the same set of tests with this model including the linear term, and again with this model including up through the cubic term, we got smaller errors but still found that there was a smooth difference curve at larger

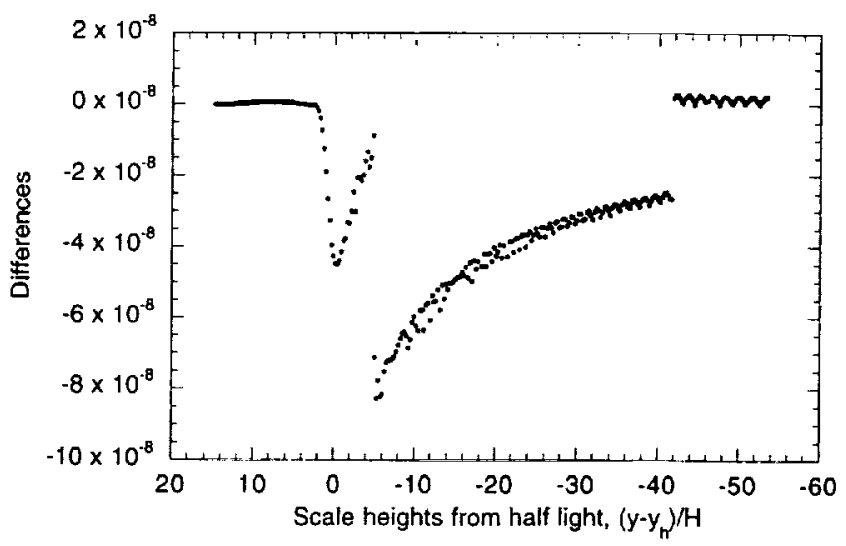

Fig. 4-Analytic test. Differences between our model results and the light curve in Fig. 3 (from the equations in Appendix (A.1) with $\lambda_{0}=100$ and $r_{h}=1500$ ). The differences are defined to be flux from the model minus the flux from our method. Note the two apparent discontinuities in the difference curve - one around five scale heights below half light, and the other around 40 scale heights below half light. We believe that they are due to numerical limitations in the implementation of our method. 


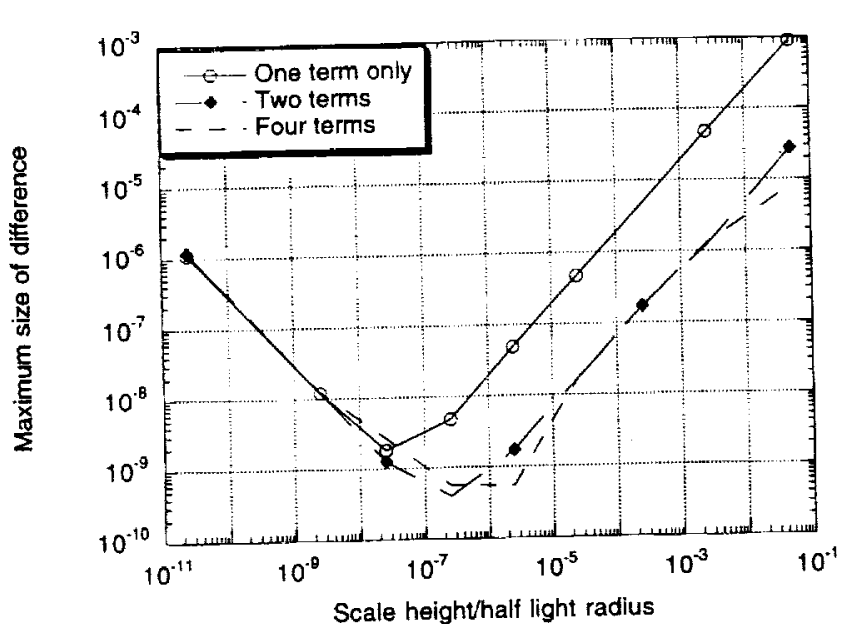

FIG. 5-Maximum size of differences in tightly bound atmosphere tests using different numbers of terms from the power series. Note that the differences in the first-order tests bottom out for $\lambda_{h}$ of about $4 \times 10^{7}$, while the tests with more terms bottom out at slightly larger ratios and generally have smaller differences, especially at smaller values of $\lambda_{h}$. The two sets of tests with more terms have similar differences, especially in the region where most real occulting bodies fall $\left(\lambda_{k}\right.$ of about $\left.10^{2}-3 \times 10^{3}\right)$.

ratios (see Fig. 7). In fact, this difference curve was almost identical for these two sets of tests.

The patterns we saw in the differences might be caused by problems with the power series. The refractivity along the path of a ray of light is Gaussian when $x$ is small compared to $r^{\prime}$, but farther out in the atmosphere where $x$ becomes comparable to $r^{\prime}$, the path of the ray becomes similar to a radial path, and the refractivity approaches a simple exponential. In the case of a large planet, this change in the behavior of $\nu$ occurs high in the atmosphere where $\nu$ is very small and thus has little effect on the bending of the ray. In smaller planets, however, this change occurs lower in the atmosphere, and could cause the differences we saw in these tests.

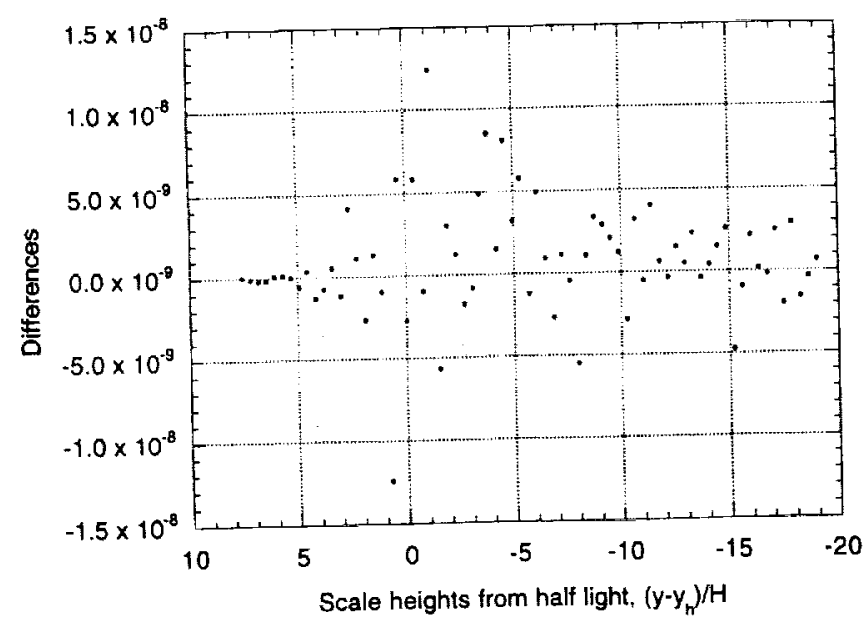

Fig. 6-Differences in one term tightly bound atmosphere test with $\lambda_{h}$ $=4 \times 10^{8}\left(r_{h}=10^{10} \mathrm{~km}, H=25 \mathrm{~km}\right)$, defined as flux from model minethod. The differences are well scattered with mean very close to zero. No pattern is visible. These differences are most likely caused by round off at the machine precision.

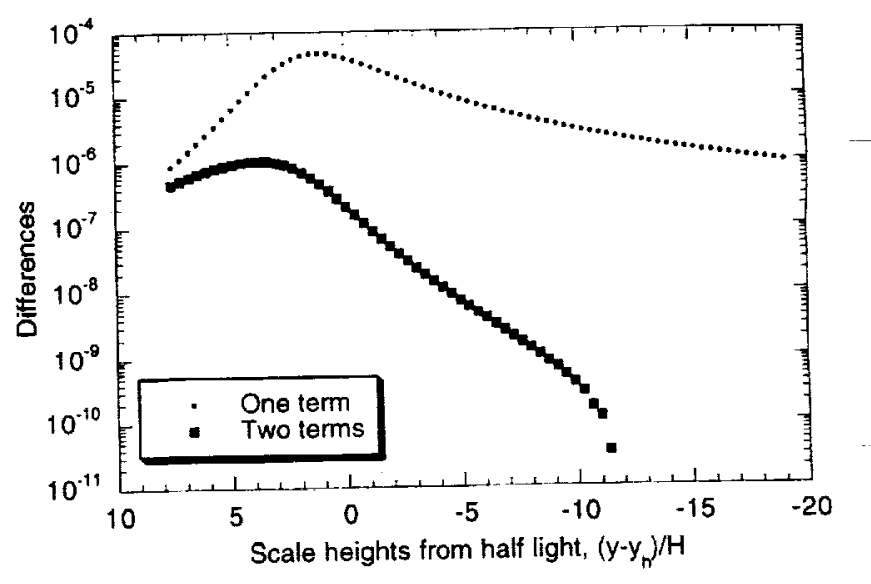

FIG. 7-Differences in one term and two term tightly bound atmosphere tests with $\lambda_{h}=4 \times 10^{3}\left(r_{h}=10^{5} \mathrm{~km}, H=25 \mathrm{~km}\right)$, as defined above. Deeper than about twelve scale heights below half light, the two term differences dropped below zero, and were of order $-10^{-11}$. The differences in both tests follow definite, smooth curves, with no evidence of scatter. However the curves have different shapes. Both of these shapes were consistent across $\lambda_{h}$ ratios, with the shape of the two term test also appearing consistently in tests where we included more than two terms.

\subsubsection{Loosely Bound Atmosphere}

Next, we ran a series of tests using EY92's isothermal model on sample planets similar to those in our solar system. Table 2 gives approximate half-light radii and scale heights for some of the planets and moons that have been observed by occultations. It shows the range of $\lambda$ values that naturally occur. We ran three groups of tests on model planets spanning the size range of the solar system: one similar to Jupiter, one similar to Venus, and one similar to Pluto.

In each group of tests, we used model planets with four different combinations of values of half-light radius $r_{h}$ and energy ratio at half-light $\lambda_{h}$. We tested how the differences depended on several properties of the profiles: how many points per scale height we had in each profile, how far above the half-light radius the profiles extended, whether or not we extended the upper boundary with the isothermal model, and how many points were calculated for the internal interpolation function for $r(y)$. We did include the

TABLE 2

Half-Light Radii and Scale Heights for Planets and Satellites in the Solar System ${ }^{\mathrm{a}}$

\begin{tabular}{lccc}
\hline \hline Planet or Satellite & $\begin{array}{c}r_{h} \\
(\mathrm{~km})\end{array}$ & $\begin{array}{c}H \\
(\mathrm{~km})\end{array}$ & $\lambda_{h}$ \\
\hline Jupiter & 71900 & 25 & 2860 \\
Saturn & 61000 & 62 & 980 \\
Venus & 6200 & 7 & 890 \\
Neptune & 25300 & 50 & 500 \\
Uranus & 26100 & 50 & 480 \\
Mars & 3500 & 8 & 430 \\
Triton & 1450 & 19 & 77 \\
Titan & 3000 & 47 & 63 \\
Pluto & 1200 & 60 & 20 \\
\hline
\end{tabular}

avalues were obtained from (Elliot and Olkin 1996) and references therein. The half-light radii are typical values for occulations observed from Earth, and scale heights are measured around the microbar pressure level in the atmosphere. 
TABI.E 3

Maximum Differences between EY92's Model and Our Method ${ }^{\mathrm{a}}$

(a) Differing tops of refractivity profiles, upper boundary extended with an isothermal model

Top of the model $\left(x_{\max }\right)$ in scale heights above half-light radius

\begin{tabular}{|c|c|c|c|c|}
\hline Model planet & 20 & 15 & 10 & 5 \\
\hline Jupiter-like & $8.5 \times 10^{-9}$ & $2.5 \times 10^{-9}$ & $4 \times 10^{-8}$ & $4 \times 10^{-6}$ \\
\hline Venus-like & $1.5 \times 10^{-8}$ & $8 \times 10^{-9}$ & $1 \times 10^{-7}$ & $9 \times 10^{-5}$ \\
\hline Pluto-like ${ }^{b}$ & $2 \times 10^{-5}$ & $3 \times 10^{-5}$ & $3 \times 10^{-4}$ & $2 \times 10^{-3}$ \\
\hline
\end{tabular}

(b) Differing tops of refractivity profiles, upper boundary not extended with an isothermal model

Top of the model $\left(x_{\max }\right)$ in scale heights above half flight radius

\begin{tabular}{cccc} 
Model planet & 20 & 15 & 10 \\
\hline Jupiter-like & $8.5 \times 10^{-9}$ & $2.2 \times 10^{-7}$ & $3.5 \times 10^{-5}$ \\
Venus-like & $1.3 \times 10^{-8}$ & $2.2 \times 10^{-7}$ & $3.1 \times 10^{-5}$ \\
Pluto-like $^{b}$ & $1.9 \times 10^{-5}$ & $6.2 \times 10^{-5}$ & $4.5 \times 10^{-4}$
\end{tabular}

(c) Differing numbers of points per scale height in the refractivity profiles

Points per scale height in refractivity profile

\begin{tabular}{ccccc} 
Model planet & 10 & 7 & 5 & 2 \\
\hline Jupiter-like & $8.5 \times 10^{-9}$ & $8.5 \times 10^{-9}$ & $8.5 \times 10^{-9}$ & $8.5 \times 10^{-9}$ \\
Venus-like & $1.3 \times 10^{-8}$ & $1.3 \times 10^{-8}$ & $1.3 \times 10^{-8}$ & $1.3 \times 10^{-8}$ \\
Pluto-like & $2.0 \times 10^{-5}$ & $2.0 \times 10^{-5}$ & $2.0 \times 10^{-5}$ & $1.9 \times 10^{-5}$
\end{tabular}

(d) Differing numbers of points used in the internal interpolation of $r(y)$

Points per scale height in refractivity profile

\begin{tabular}{llll} 
Model planet & 125 & 50 & 10 \\
\hline Jupiter-like & $8.5 \times 10^{-9}$ & $8.5 \times 10^{-9}$ & $6.8 \times 10^{-7}$ \\
Venus-like & $1.3 \times 10^{-8}$ & $1.3 \times 10^{-8}$ & $6.8 \times 10^{-7}$ \\
Pluto-like $^{\mathrm{b}}$ & $2.0 \times 10^{-5}$ & $2.0 \times 10^{-5}$ & $2.1 \times 10^{-5}$ \\
\hline \hline
\end{tabular}

${ }^{a}$ Standard conditions for the tests were (i) to extend the profiles 20 scale heights above half light, (ii) to extend the upper boundary using the isothermal atmospheric model, (iii) to have ten points per scale height in the profiles, and (iv) to calculate 125 points per scale height for $r(y)$ interpolation.

'Except for the last column, these numbers are averages of values that vary by a factor of five or more.

focusing term in these tests and ran tests along nearly central chords. For simplicity, however, we did not test regions of the light curves near the central flashes.

A summary of the results can be found in Table 3. All the results for the Jupiter- and Venus-sized model planets

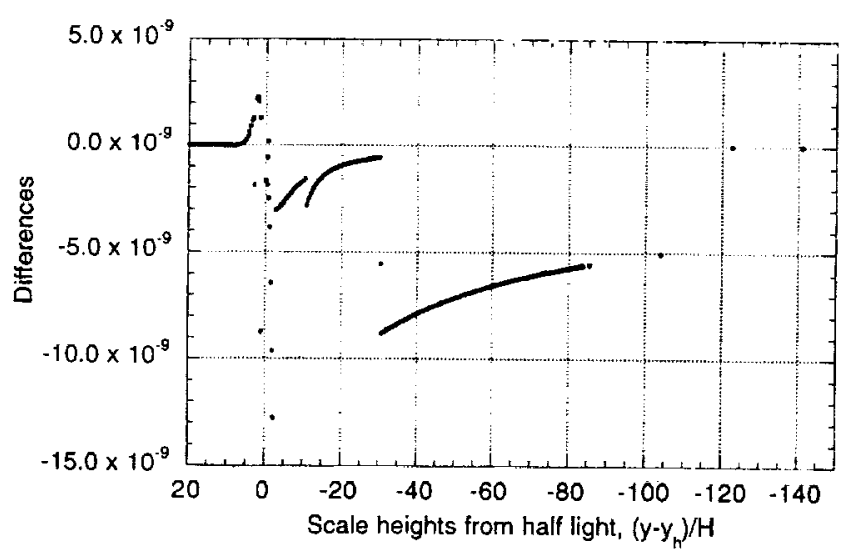

FIG. 8--A typical difference plot for a Jupiter- or Venus-sized planet test vs. the loosely bound atmosphere model. The differences are concentrated in the region where the flux is dropping off the most rapidly. We extended the light curve out to 200 scale heights above half light and down to 450 scale heights below half light, but the differences were flat and very near zero in the regions not shown. This test was run with $r_{h}=5000 \mathrm{~km}$ and $H=5 \mathrm{~km}\left(\lambda_{h}=10^{3}\right)$, and with a refractivity profile that had ten points per scale height and extended 20 scale heights above half light. We used 125 points per scale height in calculating the interpolation function for $r(y)$. (e.g., Fig. 8) were similar-none of the results were more than an order of magnitude apart. There was a weak trend for the differences in the Venus-sized cases to be larger than in the Jupiter-sized cases. In the Pluto-sized cases (e.g., Fig. 9), the differences were generally much larger and not as consistent from test to test as they were with the larger planets.

In these tests, we consistently found that the most efficient form for the profile was to have only $2-5$ points per scale height, but to have the profile extend about 15 scale heights above the half-light radius. Differences between our method and EY92's model increased sharply when we lowered the top of the profile (i.e., we decreased $x_{\max }$ ). This increase occurred more rapidly when we did not extend the upper boundary with the isothermal model than when we did. Minimum differences were achieved when we also calculated values for the interpolation of $r(y)$ at 50 or more points per scale height-much finer spacing than in the profiles.

The dependence of the size of the differences on the radius of the model planet may be explained by the power series approximation made in EY92's model. In our Plutosized test planets, we used values of 10.48 and 20.97 for $\lambda_{h}$. EY92's model includes a power series in a variable proportional to $1 / \lambda_{h}$, and we found larger differences between our method and their model in the cases where $\lambda_{h}$ was 10.48 . The size of these differences could be explained 


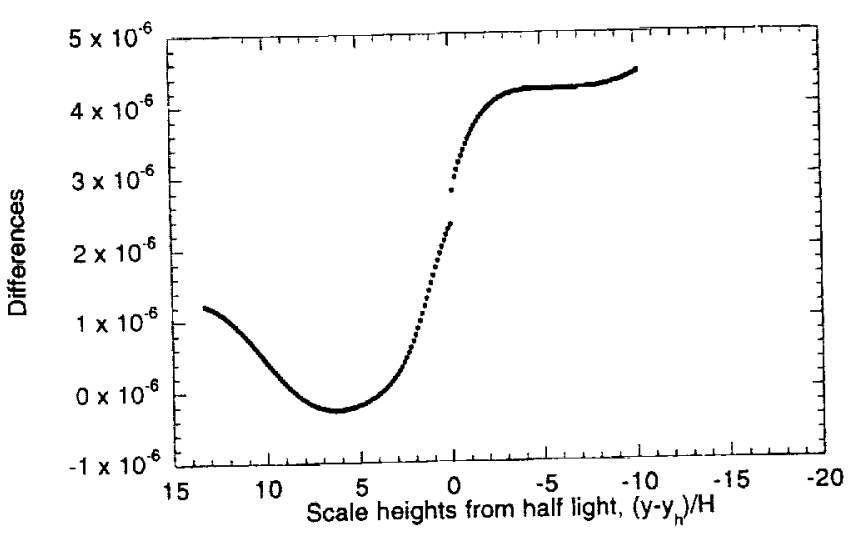

FiG. 9-A typical difference plot for a Pluto-sized planet test vs. the loosely bound atmosphere model. These differences are spread out over the entire region of the light curve, unlike the previous example. They also follow a much smoother curve. Unlike the larger planet tests, the differences from each of these small-planet tests had their own characteristic enror shape. These differences are most likely caused by approximations made in EY92's model, not by errors in our method. This test ran with $r_{h}=1250 \mathrm{~km}$ and $H=59.6 \mathrm{~km}\left(\lambda_{h}=20.8\right)$, and with a refractivity profile that had ten points per scale height and extended 20 scale heights above half light. We used 125 points per scale height in calculating the interpolation function for $r(y)$.

by the power series if the coefficient of the 5 th term in their series is of order 10 .

\subsection{Multiple Light Curves}

In this section we discuss how our method works when we interpolate between profiles and when we calculate models within a least-squares fitting procedure.

\subsubsection{Comparing Light Curves}

We ran tests in which our method interpolated between profiles to calculate the light curve. We compared EY92's no noise model for planets similar to Pluto and Triton $\left(r_{h}=1500 \mathrm{~km}, \lambda_{h}=100\right)$. For this type of planet, the errors for a single profile were around $10^{-7}$. When we ran our method with three profiles spaced $10 \%$ apart in $\lambda_{h}$ we got maximum differences between profiles of about $10^{-5}$. When we shrank the spacing to $1 \%$ and $0.01 \%$, the maximum differences were about $10^{-7}$ in both cases-the same as in the single profile case. When we ran similar tests with $r_{h}$, we found that the maximum differences between profiles were about $10^{-7}$ regardless of whether the profiles were spaced $10 \%, 1 \%$, or $0.01 \%$ apart. This difference in behavior is most likely due to the linearity of $r_{h}$-changing the half-light radius simply causes a linear shift in the light curve, while changing the energy ratio causes more complicated, nonlinear changes in the light curve. To verify this hypothesis, we compared light curves made with $10 \%$ spacing in the square root, cube root, and natural logarithm of $r_{h}$ and got slightly larger differences than we did when we used $r_{h}$ itself as the parameter.

\subsubsection{Least-Squares Fits}

Next, we used EY92's implementation of least-squares fitting in Mathematica ${ }^{\mathrm{TM}}$ to fit for $\lambda_{h}$ and $r_{h}$ in complete light curves with small central flashes. In fits for $\lambda_{h}$ and $r_{h}$ with profiles spaced $1 \%$ apart, the parameters converged to values that differed from the values used to generate the light curves by about seven parts in $10^{7}$-errors on the same order as the differences between the model light curves and those generated by our method. The errors we found when we fit both parameters simultaneously were similar to the errors we found when we fixed one parameter at the model value and fit only for the other one. Fits for $\lambda_{h}$ with profiles spaced $10 \%$ apart yielded fitted values that differed from the model parameters by four parts in $10^{5}$ when the correct model parameter values fell between profiles, and by seven parts in $10^{7}$ when the model parameters were on a profile. Similar fits for $r_{h}$ yielded errors of about seven parts in $10^{7}$ both on and off profile.

To simulate fitting real data, we also ran a series of tests on EY92's model with random Gaussian noise added to it. We characterized the noise level by specifying the ratio of the unocculted stellar signal to the rms noise level, integrated over one scale height of the atmosphere (measured in the shadow plane). We tested three different levels of noise that are typical of real data. The lowest signal-to-noise ratio per scale height used was 20; we also used values of 200 and 2000 to simulate very high signal-to-noise ratios and to study how our method performed with data of different quality. In these tests, we spaced six profiles $3 \%$ apart in the one-parameter fits and 15 profiles ( 3 in $r_{h}$ by 5 in $\lambda_{h}$ ) $8 \%$ apart in the two-parameter fits in order to have the profiles span the range of values the parameters converged to. A summary of our results is in Table 4 . Note that 28 of 42 , or $67 \%$ of our results, fell within the formal error, which is very close to the $68 \%$ expected. There appears to be a tendency for the $r_{h}$ fits to converge to a value smaller than the correct one-the fitted value was smaller than the model value in 15 of 21 tests. With this small a sample, however, the significance of this trend is difficult to determine. Statistically, if there were no bias, there would be a $4 \%$ chance of getting results at least this skewed in this direction.

As a comparison, we ran several fits of EY92's model to noisy light curves generated the same way as we did in testing our method. These fits had very similar errors to those we got with our method. Upon fitting their model to light curves fit previously by our method, we found formal errors that were the same to within $1 \%$ and fitted parameter values that were the same to within a couple percent of the formal errors.

\subsection{Computation Time}

To evaluate the speed of our method and verify that it is a faster alternative to calculating a complicated atmospheric model, we had MathematicaTM display the CPU time used in calculating the light curve each time we ran a test. For example for a Hewlett-PackardTM PA-RISC 7150 processor running at $125 \mathrm{MHz}$ (SPEC CINT95 = 4.04, CFP95 = 4.55), the one-profile EY92 tests that we ran typically took about $10 \mathrm{~min}$ of CPU time to calculate the light curve, ranging from $920 \mathrm{~s}$ down to $50 \mathrm{~s}$. In these tests, the refractivity profiles extended $5-20$ scale height above $r_{h}$ 
TABLE 4

Results of Fits to Noisy Data

\begin{tabular}{|c|c|c|c|c|c|c|c|c|c|}
\hline \multirow[b]{2}{*}{ Fit } & \multicolumn{3}{|c|}{$S / N=20$} & \multicolumn{3}{|c|}{$\mathrm{S} / \mathrm{N}=200$} & \multicolumn{3}{|c|}{$\mathrm{S} / \mathrm{N}=2000$} \\
\hline & Fitted value & $\begin{array}{c}\text { Real } \\
\text { value }\end{array}$ & $\begin{array}{c}\sigma^{\prime} \mathrm{s} \\
\text { apart }\end{array}$ & Fitted value & $\begin{array}{l}\text { Real } \\
\text { value }\end{array}$ & $\begin{array}{c}\sigma^{\prime} \mathrm{s} \\
\text { apart }\end{array}$ & Fitted value & $\begin{array}{l}\text { Real } \\
\text { value }\end{array}$ & $\underset{\text { apart }^{\mathrm{a}}}{\sigma^{\prime} \mathrm{s}}$ \\
\hline \multirow[t]{4}{*}{$\lambda_{h}$ alone } & $103.4 \pm 6.6$ & 100.5 & +0.44 & $101.92 \pm 0.62$ & 100.5 & +2.29 & $100.626 \pm 0.065$ & 100.5 & +1.93 \\
\hline & $104.1 \pm 6.8$ & 100.5 & +0.51 & $99.08 \pm 0.61$ & 100.5 & -0.34 & $100.496 \pm 0.062$ & 100.5 & -0.07 \\
\hline & $100.8 \pm 6.2$ & 101.2 & -0.07 & $102.01 \pm 0.63$ & 101.2 & +1.26 & $101.131 \pm 0.063$ & 101.2 & -1.09 \\
\hline & $95.3 \pm 5.8$ & 99.7 & -0.77 & $99.59 \pm 0.61$ & 99.7 & -0.18 & $99.623 \pm 0.061$ & 99.7 & -1.27 \\
\hline \multirow[t]{4}{*}{$r_{h}$ alone } & $1501.0 \pm 2.6$ & 1507.5 & -2.51 & $1507.11 \pm 0.26$ & 1507.5 & -1.51 & $1507.512 \pm 0.026$ & 1507.5 & +0.48 \\
\hline & $1504.9 \pm 2.6$ & 1507.5 & -0.98 & $1507.00 \pm 0.25$ & 1507.5 & -2.01 & $1507.488 \pm 0.026$ & 1507.5 & -0.46 \\
\hline & $1519.9 \pm 2.5$ & 1521.0 & -0.42 & $1520.82 \pm 0.26$ & 1521.0 & -0.68 & $1520.976 \pm 0.023$ & 1521.0 & -0.96 \\
\hline & $1497.2 \pm 2.6$ & 1498.0 & -0.32 & $1498.09 \pm 0.25$ & 1498.0 & +0.38 & $1498.003 \pm 0.025$ & 1498.0 & +0.10 \\
\hline \multirow[t]{2}{*}{ both, $\lambda_{h}$} & $109.1 \pm 7.4$ & 100.5 & +1.17 & $99.52 \pm 0.62$ & 100.5 & -1.59 & $100.501 \pm 0.066$ & 100.5 & +0.01 \\
\hline & $109.9 \pm 7.2$ & 101.2 & +1.20 & $101.20 \pm 0.65$ & 101.2 & 0.00 & $101.159 \pm 0.063$ & 101.2 & -0.64 \\
\hline an. & $99.7 \pm 6.7$ & 99.7 & 0.00 & $99.59 \pm 0.64$ & 99.7 & -0.17 & $99.718 \pm 0.062$ & 99.7 & +0.29 \\
\hline \multirow[t]{3}{*}{ both, $r_{h}$} & $1508.1 \pm 2.5$ & 1507.5 & +0.20 & $1507.74 \pm 0.24$ & 1507.5 & +1.03 & $1507.491 \pm 0.026$ & 1507.5 & -0.36 \\
\hline & $1519.1 \pm 2.4$ & $152 \mathrm{~L} .0$ & -0.79 & $1520.70 \pm 0.26$ & 1521.0 & -1.18 & $1520.992 \pm 0.025$ & 1521.0 & -0.33 \\
\hline & $1494.4 \pm 2.7$ & 1498.0 & -1.33 & $1520.70 \pm 0.26$ & 1498.0 & -0.32 & $1498.025 \pm 0.025$ & 1498.0 & +0.98 \\
\hline
\end{tabular}

"These numbers are (fitted value-real value)/(standard deviation of fitted value).

with $2-10$ points per scale height, and we calculated $10-$ 125 points per scale height in the internal interpolation function for $r(y)$ and 200-700 points in the final light curve. The fastest tests were the ones where we did not calculate as many points for the internal interpolation of $r(y)$. Extending the upper boundary with the isothermal model generally added about $25 \%$ to the calculation time. Using refractivity profiles with fewer points per scale height had little effect on the calculation time for the model, but lowering the top of the profile (decreasing $x_{\max }$ ) increased the time considerably. The number of points at which we wished to calculate the light curve did not seem to be a major factor. Our tests often had different numbers of points in them, and some of the tests with more points in the light curve ran faster than tests with fewer.

The time needed for least-squares fits depends on the number of parameters fit and the number of iterations needed for the values of the particular set of parameters to converge. In general, to determine the time needed for a fit, one should multiply the times given above by the number of parameters fit, and multiply that by the number of iterations needed. Our fits for two parameters often took as long as 3-4 h. If we implemented this method in $\mathrm{C}$, we predict that such two parameter fits would take only about $2 \mathrm{~min}$.

\section{CONCLUSIONS}

We have developed a method for numerically calculating occultation light curves from an arbitrary atmospheric model that is fast enough to enable least-squares fitting for model parameters. This method can be applied to a broad range of bodies, including Pluto and Triton. We have tested and verified that our method shows reasonable agreement with more exact calculations. Small, systematic errors in our method (most likely due to numerical procedures) are at a level much lower than the noise levels in recent occultation observations. To date, the best signal-to-noise per scale height levels have been on the order of $10^{3}$. When we used sufficiently complete refractivity profiles, the largest differences between our method and the comparison models were on the order of $10^{-4}$.

We found that it is better to interpolate the refractivity derivatives than to interpolate light curves, and that the most efficient form for refractivity profiles that still gave good results was for each profile to extend well above the half-light radius but to include only a few points per scale height. The quality of results obtained with such profiles implies that, if necessary, it is acceptable to record occultation data with as few as two light-curve points per scale height.

Planned improvements to our procedure include speeding up the calculations by implementing the code in a compiled language, such as $\mathrm{C}$. This will enable the least-squares fitting of atmospheric models with more parameters. Also, for large, oblate planets-which do not satisfy the assumption of spherical symmetry-one could recast the fundamental equations in terms of local radii of curvature, rather than the single radius used for the spherical-symmetry assumption.

We thank Catherine Olkin for interesting discussions, helping us debug our code, and providing incentive for us to get it working quickly when she needed it for her thesis. We also are grateful to Bill Hubbard for his comments as referee and appreciate suggestions from Mary Agner. This work was supported, in part, by NASA Grant NAGW-1494, NSF Grant AST-9322115, and NSF's Research Experiences for Undergraduates (REU) Program.

\section{APPENDIX: EQUATIONS USED FOR MODEL- ATMOSPHERE TESTS}

In this Appendix we develop the equations in the form needed for the tests of our method performed on an analytic atmosphere and a tightly bound atmosphere. Equations for the loosely bound atmosphere appear in EY92. 


\section{A.1 Analytic Atmosphere}

Eshleman and Gurrola (1993) have presented a set of descriptive atmospheric models that satisfy hydrostatic equilibrium for different power-law radial dependencies of gravity and temperature. For a spherically symmetric gravity field (i.e., $g(r)=G M / r^{2}$ ), an inverse radial temperature dependence (i.e., $T(r)=T_{0} r_{0} / r$, where $r_{0}$ is a reference radius) yields a model expressible with ordinary functions.

If $H_{0}$ is the pressure scale height at the reference radius, we define $\lambda_{0} \equiv r_{0} / H_{0}$. The refractivity and its first two derivatives for this analytic model, needed to calculate the light curve with our method, are given by

$$
\begin{gathered}
\nu(r)=\nu_{0}\left(\frac{r}{r_{0}}\right)^{-\left(\lambda_{0}-1\right)} \\
\frac{d \nu(r)}{d r}=-\frac{\nu_{0}\left(\lambda_{0}-1\right)}{r_{0}}\left(\frac{r}{r_{0}}\right)^{-\lambda_{0}} \\
\frac{d^{2} \nu(r)}{d r^{2}}=\frac{\nu_{0} \lambda_{0}\left(\lambda_{0}-1\right)}{r_{0}^{2}}\left(\frac{r}{r_{0}}\right)^{-\left(\lambda_{0}+1\right)} .
\end{gathered}
$$

We also need the analytic expressions for the refraction angle and its derivative, required for calculating an analytic light curve (Eshleman and Gurrola 1993):

$$
\theta(r)=-\frac{2 \nu_{0} \pi^{1 / 2} \Gamma\left(\frac{\lambda_{0}}{2}\right)}{\Gamma\left(\frac{\lambda_{0}}{2}-\frac{1}{2}\right)}\left(\frac{r}{r_{0}}\right)^{-\left(\lambda_{0}-1\right)} .
$$

Its derivative is

$$
\frac{d \theta(r)}{d r}=\frac{2 \nu_{0} \pi^{1 / 2}\left(\lambda_{0}-1\right) \Gamma\left(\frac{\lambda_{0}}{2}\right)}{r_{0} \Gamma\left(\frac{\lambda_{0}}{2}-\frac{1}{2}\right)}\left(\frac{r}{r_{0}}\right)^{-\lambda_{0}} .
$$

The value of the reference radius, $r_{0}$, is arbitrary, so we choose to set it to the half-light radius, $r_{h}$. To find $r_{h}$, we set $\phi_{\text {cyl }}$ to 0.5 in Eq. (4) and solve for $d \theta / d r$. Substituting this expression into Eq. (A1.5) and solving for $r_{0}$ gives us the following formula for $r_{h}$ :

$$
r_{0}=r_{h}=\frac{2 \nu_{0} \pi^{1 / 2} D\left(\lambda_{0}-1\right) \Gamma\left(\frac{\lambda_{0}}{2}\right)}{\Gamma\left(\frac{\lambda_{0}}{2}-\frac{1}{2}\right)},
$$

then, by definition, $\lambda$ at half-light, $\lambda_{h}=\lambda_{0}$. To calculate the analytic light curve, we substitute Eq. (A1.6) for $r_{0}$ in Eq. (AI.5) and then put this equation into Eq. (4). Equations (5) and (A1.4) can be used to relate $r$ and $y$.

\section{A.2 Tightly Bound Atmosphere}

In the exact equation for a cylindrical atmosphere (one with no focusing in the plane perpendicular to the ray path), the light-curve flux, $\phi_{\text {cyl }}$, is related to the derivative of the refraction angle, $\theta(r)$ for an atmosphere at a distance $D$ by Eq. (4):

$$
\phi_{\mathrm{cyl}}=\frac{1}{\left|1+D \frac{d \theta}{d r}\right|} .
$$

Also, $y$ is related to the refraction angle in the small-angle approximation by Eq. (5):

$$
y(r)=r+D \theta(r) \text {. }
$$

If we pick a reference ray that intercepts the atmosphere at a radius $r_{0}$ and is then refracted by the atmosphere by an angle $\theta_{0}$, so that it intercepts the observer at $y_{0}$, we have

$$
y_{0}=r_{0}+D \theta_{0} .
$$

We postulate that the atmosphere produces an exponential refraction angle with a scale height $H$, so that

$$
\theta(r)=\theta_{0} e^{-\left(r-r_{0}\right) / H} .
$$

Next, we subtract (A3) from (A2) and divide by the scale height $H$ :

$$
\frac{\left(y-y_{0}\right)}{H}=\frac{\left(r-r_{0}\right)}{H}+\frac{D}{H}\left(\theta-\theta_{0}\right) .
$$

We choose to put the reference level at half light $\left(\phi_{c y l}=1 / 2\right)$. Now we can solve for $\theta_{0}$ :

$$
\phi_{\mathrm{cyl}}=\frac{1}{2}=\left.\frac{1}{\left|1+D \frac{d \theta}{d r}\right|}\right|_{r=r_{0}}=\frac{1}{1-\frac{D \theta_{0}}{H}},
$$

or

$$
\theta_{0}=-\frac{H}{D}
$$

Substituting $\theta_{0}=-H / D$ in to the flux equation (Eq. (4)), we get

$$
\phi_{\mathrm{cyl}}=\frac{1}{\left|1+D \frac{d \theta}{d r}\right|}=\frac{1}{\left|1+e^{-\left(r-r_{0}\right) / H}\right|}=\frac{1}{1+e^{-\left(r-r_{0}\right) / H}} .
$$

Solving for the exponential in terms of the flux, we find

$$
e^{-\left(r-r_{0}\right) / H}=\frac{1}{\phi_{\text {cyl }}}-1 .
$$

The exponent is

$$
\frac{\left(r-r_{0}\right)}{H}=-\ln \left(\frac{1}{\phi_{\text {cyl }}}-1\right),
$$

which is the first term on the right-hand side of Eq. (A2.5). Next we look at the second term on the right-hand side of Eq. (A2.5):

$$
\begin{aligned}
\frac{D}{H}\left(\theta-\theta_{0}\right)=\frac{D \theta}{H}-\frac{D \theta_{0}}{H} & =\frac{D \theta_{0}}{H}\left[e^{-\left(r-r_{0}\right) / H}-1\right] \\
& =\frac{1}{\phi_{\text {cyl }}}-2,
\end{aligned}
$$


where the last step in Eq. (A2.11) used Eqs. (A2.4) and (A2.9). Substituting this result and Eq. (A2.10) into Eq. (A2.5) and denoting this is the observer's $y$ coordinate at half light by $y_{h}$, we find

$$
\frac{y(t)-y_{h}}{H}=-\ln \left(\frac{1}{\phi_{\mathrm{cyl}}(y)}-1\right)-\left(\frac{1}{\phi_{\mathrm{cyl}}(y)}-2\right) \text {. }
$$

This light-curve equation, valid for exact exponential refraction, is identical to the approximation derived by Baum and Code (1953) for the occultation light curve for a large, spherical planet with an isothermal atmosphere.
Next, we need a formula for the refractivity, $\nu(r)$, that yields an exponential $\theta(r)$ given by Eq. (A2.4). To find the desired function we first tried a simple exponential and calculated the refraction angle $\theta$ for this $\nu$. We found that $\theta$ would be exponential to first order if we multiplied the exponential by $1 / \sqrt{r}$ :

$$
\nu_{1}(r)=\nu_{h} \sqrt{\frac{r_{h}}{r}} e^{-\left(r-r_{h}\right) / H} .
$$

To make $\theta$ be exponential to higher order, we multiplied $\nu_{1}$ by a power series in $H / r$, choosing the coefficients to cancel out the nonexponential terms in $\theta(r)$ :

$$
\nu(r)=\nu_{h} \sqrt{\frac{r_{h}}{r}} e^{\left(r-r_{h}\right) / H}\left[1-\frac{1}{8}\left(\frac{H}{r}\right)+\frac{9}{128}\left(\frac{H}{r}\right)^{2}-\frac{75}{1024}\left(\frac{H}{r}\right)^{3}+\frac{3675}{32768}\left(\frac{H}{r}\right)^{4}-\frac{59535}{262144}\left(\frac{H}{r}\right)^{5}+\cdots\right] .
$$

Note that the coefficients begin to slowly increase in size, indicating that this series is asymptotic and will most likely diverge if we include enough terms.

\section{REFERENCES}

Baum, W. A., and Code, A. D. 1953, AJ, 58, 108

Born, M., and Wolf, E. 1964, Principles of Optics (New York, MacMillan)

Chamberlain, D. M. 1996, A Numerical Method for Calculating Occultation Light Curves from an Arbitrary Atmospheric Model, S.B. thesis, Massachusetts Institute of Technology

Elliot, J. L., et al. 1993, AJ, 106, 2544

Elliot, J. L., French, R. G., Dunham, E., Gierasch, P. J., Veverka, J., Church, C., and Sagan, C. 1977, ApJ, 217, 661

Elliot, J. L., and Olkin, C. B. 1996, in Annual Review of Earth and Planetary Sciences, ed. G. W. Wetherill (Palo Alto, Annual
Reviews), p. 89

Elliot, J. L., and Young, L. A. 1992, AJ, 103, 991

Eshleman, V. R., and Gurrola, E. M. 1993, Icarus, 105, 298

French, R. G., Elliot, J. L., and Gierasch, P. J. 1978, Icarus, 33, 186

Kovalevsky, J., and Link, F. 1969, A\&A, 2, 398

Krasnopolsky, V. A., Sandel, B. R., Herbert, F., and Vervack, R. J. 1993, JGR, 98, 3065

Olkin, C. B. 1996, Ph.D. thesis, Massachusetts Institute of Technology

Roques, F., et al. 1994, A\&A, 288, 985

Strobel, D. F., and Summers, M. E. 1995, in Neptune and Triton, ed. D. P. Cruikshank (Tucson, University of Arizona Press), p. 1107

Wasserman, L. H., and Veverka, J. 1973, Icarus, 20, 322

Wolfram, S. 1991, Mathematica (Redwood City, CA, AddisonWesley) 
$\overline{8}$ 